\title{
CONSTANT MEAN CURVATURE SURFACE, HARMONIC MAPS, AND UNIVERSAL TEICHMÜLLER SPACE
}

\author{
TOM Y. H. WAN
}

\section{Introduction}

It has been known for some years that the Gauss map of a space-like surface in Minkowski 3-space $\mathbf{M}^{2,1}$ is harmonic if and only if the mean curvature of the surface is constant (see [7]). Using this fact, we can construct, for each holomorphic quadratic differential on a simply-connected domain $\Omega$ in $\mathbf{C}$, an injective harmonic map from $\Omega$ into the Poincaré disk $\mathbf{D}$. This harmonic map is unique up to equivalent classes, provided that it satisfies a completeness condition. In the process, we will find a classification of all complete hyperbolic space-like surfaces of constant mean curvature in $\mathbf{M}^{2,1}$.

It is also known that harmonic maps between surfaces are deeply related to the Teichmüller theory of compact Riemann surfaces (see [9]). So, it is interesting to study the analogy for universal Teichmüller space. For this problem, we find that a harmonic diffeomorphism from $\mathbf{D}$ onto itself is quasi-conformal if and only if the associated Hopf differential is bounded with respect to the Poincare metric. From this result, we have a continuous map from the space of equivalence classes of holomorphic quadratic differentials under the action of the Möbius group, which is bounded with respect to the Poincaré metric, into the universal Teichmüller space.

The author would like to thank Professor R. Schoen for his continuous encouragement and support during the last few years. Without his support, this paper would not have been possible.

\section{Geometric preliminaries}

The Minkowski 3-space $\mathbf{M}^{2,1}$ is $\mathbf{R}^{2} \times \mathbf{R}^{1}$ endowed with the metric

$$
d s^{2}=\left(d x^{1}\right)^{2}+\left(d x^{2}\right)^{2}-\left(d x^{3}\right)^{2},
$$

Received January 15, 1991. 
where $x=\left(x^{1}, x^{2}\right)$ and $x^{3}$ are Euclidean coordinates of $\mathbf{R}^{2}$ and $\mathbf{R}^{1}$, respectively.

Let $S$ be a space-like surface in $\mathbf{M}^{2,1}$, that is, $S$ be a surface whose induced metric is Riemannian. Then, locally, $S$ is given as a graph of a function $x^{3}=f\left(x^{1}, x^{2}\right)$ satisfying the space-like condition $|D f|<1$. As in the case of a surface in Euclidean 3-space $\mathbf{R}^{3}$, we can define the first and the second fundamental forms of $S$ as follows:

$$
\mathrm{I}=g_{i j} d x^{i} d x^{j}, \quad \mathrm{II}=h_{i j} d x^{i} d x^{j} .
$$

The mean curvature $H$ and the Gaussian curvature $K$ are given by

$$
2 H=\frac{h_{22} g_{11}-2 h_{12} g_{12}+h_{11} g_{22}}{\operatorname{det}\left(g_{i j}\right)}, \quad K=-\frac{\operatorname{det}\left(h_{i j}\right)}{\operatorname{det}\left(g_{i j}\right)} .
$$

Note that $K=\operatorname{det}\left(h_{i j}\right) / \operatorname{det}\left(g_{i j}\right)$ in the Euclidean case (see [7]).

For a space-like surface $S$ in $\mathbf{M}^{2,1}$, there is a natural projection $\Pi: S \rightarrow$ $\mathbf{R}^{2}$ defined by $\Pi\left(x^{1}, x^{2}, x^{3}\right)=\left(x^{1}, x^{2}\right) \in \mathbf{R}^{2}$ for any $\left(x^{1}, x^{2}, x^{3}\right) \in S$. If $\Pi$ is onto $\mathbf{R}^{2}$, we say that $S$ is entire.

Proposition 1. Let $\Omega$ be a domain in $\mathbf{C}$ and let $S: \Omega \rightarrow \mathbf{M}^{2,1}$ be a space-like immersion. Suppose that $S$ is complete with respect to the induced Riemannian metric. Then $S$ is an entire graph and $\Omega$ is simplyconnected.

Proof. Suppose that $S$ is not entire. Then $\partial \Pi(S)$ is nonempty in C. So we can find a smooth arc $\gamma:[0,1] \rightarrow \overline{\Pi(S)}$ with finite Euclidean length such that $\gamma[0,1) \subset \Pi(S)$ and $\gamma(1) \in \partial \Pi(S)$.

Let $z$ be a point in $\Omega$ such that $\Pi \circ S(z)=\gamma(0)$. Since $S$ is a spacelike immersion, $\Pi \circ S$ is a local homeomorphism. Therefore, we can lift a portion of $\gamma$, starting from $\gamma(0)$, up to a path $\gamma^{*}$ on $\Omega$. Suppose that $\bar{t}$ is the maximal $t \in[0,1]$ such that we can lift $\gamma$. Since $\Pi \circ S$ is a local homeomorphism, either $\lim _{t \rightarrow \bar{t}} \gamma^{*}(t)$ exists or the set of limiting points contains in $\partial \Omega$. Since the length of $\gamma^{*}$ with respect to the induced metric is bounded by the Euclidean length of $\gamma$, the latter case contradicts the completeness of $S$. Similarly, $\lim _{t \rightarrow \bar{t}} \gamma^{*}(t)$ must belong to the interior of $S$. So we can lift $\gamma$ for further $t$. Therefore $\bar{t}=1$ and $\lim _{t \rightarrow 1} \gamma^{*}(t)$ exists and belongs to the interior of $S$. This contradicts the assumption that $\gamma(1) \in \partial \Pi(S)$. Hence, $S$ is entire.

Suppose $\Pi \circ S$ is not one-one. Let $z_{1}$ and $z_{2}$ be two points in $\Omega$ such that $\Pi \circ S\left(z_{1}\right)=\Pi \circ S\left(z_{2}\right)=w$. Take a path $\sigma^{*}$ in $\Omega$ with endpoints equal to $z_{1}$ and $z_{2}$. Then $\sigma=\Pi \circ S \circ \sigma^{*}$ is a closed path, which may not be simple, in $\mathbf{C}$. Using the fact that $\mathbf{C}$ is simply-connected, we have a homotopy shrinking $\sigma$ to the point $w$. Since $\sigma^{*}$ is compact and $\Pi \circ S$ 
is a local homeomorphism, we can lift the homotopy locally near $\sigma^{*}$. In fact, we can lift the homotopy until we hit a branch point or the boundary. On the one hand, we have no branch point because $\Pi \circ S$ is locally homeomorphic. On the other hand, we cannot hit the boundary because of the completeness of $S$. Therefore, we can lift the homotopy. But then we have a path on $\Omega$ with endpoints $z_{1}, z_{2}$ which completely lies in $(\Pi \circ S)^{-1}(w)$. This contradicts the fact that $\Pi \circ S$ is locally homeomorphic. So $\Pi \circ S$ is one-one and hence $S$ is a graph. The final conclusion is now obvious. q.e.d.

From Lemma 1 and a result in [4], we have the following:

Proposition 2. Let $S$ be a complete space-like surface in $\mathbf{M}^{2,1}$. Suppose that the mean curvature $H$ of $S$ is constant. Then $S$ can be represented as a graph of a function $x^{3}=f(x)=f\left(x^{1}, x^{2}\right)$ such that $f$ is defined for all $x \in \mathbf{R}^{2}$ and satisfies the equation

$$
\sum_{i} \frac{\partial}{\partial x^{i}}\left(\frac{f_{x^{i}}}{\sqrt{1-|D f|^{2}}}\right)=2 H .
$$

Moreover, $f$ is a convex function, and $S$ has nonpositive Gaussian curvature.

Proof. By Lemma 1, we conclude that $S$ is entire and closed with respect to the Euclidean topology of $\mathbf{R}^{3}$. Then the results follow from [4] (see also [5]). q.e.d.

We say that $S$ is a constant mean curvature cut if $S$ is an entire spacelike surface in $\mathbf{M}^{2,1}$ with constant mean curvature $H>0$, and if it is complete. Without loss of generality, we assume $H=1$ throughout this paper. We end this section by mentioning that the Gauss map of a spacelike surface in $\mathbf{M}^{2,1}$ is harmonic into the hyperbolic 2-space if and only if the mean curvature is constant (see [7], [5]).

\section{Harmonic maps between surfaces}

Let $\Sigma_{1}$ and $\Sigma_{2}$ be two surfaces. If $\rho^{2}(z)|d z|^{2}$ and $\sigma^{2}(u)|d u|^{2}$ are the metrics with respect to isothermal coordinate charts on $\Sigma_{1}$ and $\Sigma_{2}$, respectively, then $u: \Sigma_{1} \rightarrow \Sigma_{2}$ as a harmonic map satisfies

$$
u_{z \bar{z}}+\frac{2 \sigma_{u}}{\sigma} u_{z} u_{\bar{z}}=0
$$


and the $\partial$ and $\bar{\partial}$ energy densities are given by

$$
|\partial u|^{2}=\frac{\sigma^{2}}{\rho^{2}}\left|u_{z}\right|^{2}, \quad|\bar{\partial} u|^{2}=\frac{\sigma^{2}}{\rho^{2}}\left|u_{\bar{z}}\right|^{2} .
$$

We have

Lemma 3. At points where $\partial u(\bar{\partial} u$, respectively) is nonzero we have

$$
\begin{gathered}
\Delta_{\Sigma_{1}} \log |\partial u|^{2}=-2 K_{2} J(u)+2 K_{1}, \\
\left(\Delta_{\Sigma_{1}} \log |\bar{\partial} u|^{2}=2 K_{2} J(u)+2 K_{1}\right),
\end{gathered}
$$

where $K_{i}$ denotes the Gaussian curvature of $\Sigma_{i}$, and $J(u)=|\partial u|^{2}-|\bar{\partial} u|^{2}$ is the Jacobian of $u$.

Proof. See [8].

In this paper, $\Sigma_{2}$ will always be the Poincaré disk $\left(\mathbf{D}, d s_{p}^{2}\right)$, where

$$
d s_{p}^{2}=\frac{4|d z|^{2}}{\left(1-|z|^{2}\right)^{2}}
$$

so we have $K_{2}=-1$. We will also restrict ourself to those harmonic maps which satisfy $J(u) \geq 0$ and $|\partial u|^{2}>0$. This is exactly the case when $u$ is coming from a Gauss map of a constant mean curvature space-like surface. In this case, $w=\frac{1}{2} \log |\partial u|^{2}$ is a well-defined function on $\Sigma_{1}$.

It is also well known that the quadratic differential

$$
\Phi(z)=\phi(z) d z^{2},
$$

where $\phi(z)=\sigma^{2}(u(z)) u_{z} \bar{u}_{z}$, is holomorphic; that is, $\phi(z)$ is a holomorphic function on $\Sigma_{1}$. If $|\partial u|^{2}>0$, we can write

$$
|\bar{\partial} u|^{2}=\rho^{4}(z)|\phi(z)|^{2}|\partial u|^{-2}=\|\Phi\|^{2}(z)|\partial u|^{-2},
$$

where $\|\Phi\|^{2}(z)=\rho^{-4}(z)|\phi(z)|^{2}$ is independent of the particular isothermal coordinates. Therefore the first equation in Lemma 3 can be rewritten as

$$
\Delta_{\Sigma_{1}} w=e^{2 w}-\|\Phi\|^{2} e^{-2 w}+K_{1} .
$$

Note that we have already used the assumption that $\Sigma_{2}$ is the Poincare disk.

In $\S 2$, we mentioned that the Gauss map of a space-like constant mean curvature surface $S$ in $\mathbf{M}^{2,1}$ is a harmonic map into the Poincaré disk. The uniformization theorem implies that $S$ is conformally equivalent to either $\left(\mathbf{C},|d z|^{2}\right)$ or $\left(\mathbf{D}, d s_{p}^{2}\right)$. Therefore, we can represent $S$ as $\left(\mathbf{C}, g_{1}\right)$ or $\left(\mathbf{D}, g_{2}\right)$, where the $g_{i}$ are conformally equivalent to 
the metric $|d z|^{2}$, and the composition of the Gauss map and the conformal parametrization $u: \Sigma_{1} \rightarrow\left(\mathbf{D}, d s_{p}^{2}\right)$ is harmonic, where $\Sigma_{1}$ is either $\left(\mathbf{C},|d z|^{2}\right)$ or $\left(\mathbf{D}, d s_{p}^{2}\right)$. The important observation is

Proposition 4. Suppose that the mean curvature of $S$ is constantly one. Then the induced metric $g$ on $S$ and the Gaussian curvature $K$ of $S$ are given by

$$
g=\frac{4}{\left(1-|u|^{2}\right)^{2}}\left|u_{z}\right|^{2}|d z|^{2}, \quad K=\left|\frac{u_{\bar{z}}}{u_{z}}\right|^{2}-1 .
$$

Proof. See [2] or [1].

Since the harmonicity depends only on the conformal class of the metric on $\Sigma_{1}$, we can choose any metric in the conformal class which is convenient to us. In the case where the metric on $\Sigma_{1}$ is given by $g_{0}=\rho^{2}|d z|^{2}$, the induced metric on $S$ is given by

$$
g=|\partial u|^{2} \rho^{2}|d z|^{2}=|\partial u|^{2} g_{0} \text {. }
$$

Remark. From the proposition, we see that if a harmonic map $u$ is given by a Gauss map of a constant mean curvature cut, then $|\partial u|^{2}>0$ and $J(u)=|\partial u|^{2}(-K) \geq 0$, since $K \leq 0$ by Proposition 2 .

\section{Classification of hyperbolic constant mean curvature cuts}

Let $S$ be a hyperbolic constant mean curvature cut. Then there exists a conformal diffeomorphism from $\mathbf{D}$ onto $S$, and we can consider the Gauss map of $S$ as a harmonic map from the Poincaré disk into itself. Therefore, we can associate a holomorphic quadratic differential on $\mathbf{D}$ for each $S$. Notice that the differential depends only on the equivalent class of isometric surfaces of $S$, but not on $S$ itself. Hence, we have a well-defined map $\mathscr{A}$ from the set $\mathbf{E}$ of equivalent classes of hyperbolic constant mean curvature cuts into the space QD of equivalent classes of holomorphic quadratic differentials on $\mathbf{D}$ under the action of the Möbius group (this corresponds to change of conformal parameters on $S$.) We will show that $\mathscr{A}$ is actually one-one and onto.

Theorem 5. $\mathscr{A}: \mathbf{E} \rightarrow \mathbf{Q D}$ is one-one and onto.

Let $S$ be a representative of an element in $\mathbf{E}, u$ be the harmonic map associated to $S$, and $\Phi(z)=\phi(z) d z^{2}$ be its Hopf differential. Then

$$
w=\frac{1}{2} \log |\partial u|^{2}
$$

is a well-defined function on $\mathbf{D}$, and $e^{2 w} d s_{p}^{2}$ is the induced metric on $S$. 
The harmonicity of $u$ yields that $w$ and $\|\Phi\|$ satisfy

$$
\Delta_{p} w=e^{2 w}-\|\Phi\|^{2} e^{-2 w}-1
$$

Moreover, the assumption that $S$ is complete implies that $\left(\mathbf{D}, e^{2 w} d s_{p}^{2}\right)$ is a complete Riemannian manifold.

Conversely, if we can uniquely solve a solution $w$ for a given $\|\Phi\|$, then we can use the following equations to solve for $h_{i j}$ :

$$
h_{11}+h_{22}=2 e^{2 w} \frac{4}{\left(1-|z|^{2}\right)^{2}}, \quad h_{11}-h_{22}=2 \mathfrak{R}(\phi), \quad h_{12}=-\mathfrak{I}(\phi),
$$

where $\mathfrak{R}(\phi)$ and $\mathfrak{I}(\phi)$ are the real and imaginary parts of $\phi$, respectively. Using those $h_{i j}$, we can construct two two-forms which satisfy the Gauss and Codazzi equations. Namely, if

$$
\mathrm{I}=e^{2 w} d s_{p}^{2}, \quad \mathrm{II}=h_{i j} d x^{i} d x^{j},
$$

then the Gauss equation is translated to (1), where the fact that $\Phi$ is holomorphic is equivalent to the Codazzi equations being satisfied. Therefore, by the fundamental theorem of differential geometry, we can construct locally a space-like surface, unique up to Lorentz motions, in $\mathbf{M}^{2,1}$ with first and second fundamental forms equal to I and II, respectively. Since D is simply-connected, we actually have a global solution surface $S$. By construction, $S$ has constant mean curvature one. If $e^{2 w} d s_{p}^{2}$ is complete, then by Proposition $2, S$ is a complete hyperbolic constant mean curvature cut. Finally, by Proposition 4, the holomorphic quadratic differential associated to $S$ is exactly given by $\Phi$. Therefore, the map $\mathscr{A}$ being one-one onto is equivalent to the uniqueness and existence of $w$ for the equation

$$
1+\Delta_{p} w=e^{2 w}-\|\Phi\|^{2} e^{-2 w},
$$

such that $e^{2 w} d s_{p}^{2}$ is complete and $e^{2 w}-\|\Phi\|^{2} e^{-2 w} \geq 0$.

\section{Uniqueness}

For the uniqueness of (2), we will prove a slightly more general theorem.

Theorem 6. Let $h$ be a continuous function defined on a domain $\Omega \subset$ $\mathbf{C}$, and let $\tilde{w}$ be a solution of

$$
\Delta \tilde{w}=e^{2 \tilde{w}}-h^{2} e^{-2 \tilde{w}} \geq 0
$$

in $\Omega$ such that $e^{2 \tilde{w}}|d z|^{2}$ is a complete Riemannian metric on $\Omega$. Then, for any other solution $v$ of the equation, $v \leq \tilde{w}$. 
Proof. Let $\eta=v-w$. Then

$$
\Delta \eta=e^{2 v}-h^{2} e^{-2 v}-e^{2 \tilde{w}}+h^{2} e^{-2 \tilde{w}},
$$

which implies

$$
\Delta_{g} \eta=e^{2 \eta}-|\mu|^{2} e^{-2 \eta}-1+|\mu|^{2},
$$

where $\Delta_{g}$ is the Laplacian on $\Omega$ with respect to the metric $g=e^{2 \tilde{w}}|d z|^{2}$, and $|\mu|^{2}=h^{2} e^{-4 \tilde{w}}$. Since $e^{2 \tilde{w}}-h^{2} e^{-2 \tilde{w}} \geq 0$, we have $|\mu|^{2} \leq 1$. Therefore

$$
\Delta_{g} \eta \geq e^{2 \eta}-e^{-2 \eta}-1 \text {. }
$$

A result in [3] shows that $\eta$ is bounded from above, provided that $g$ is complete and its curvature is bounded from below. The metric $g$ is assumed to be complete, so we only need to check that the Gaussian curvature $K(g)$ is bounded from below. In fact

$$
K(g)=-\frac{\Delta \log e^{2 \tilde{w}}}{2 e^{-2 \tilde{w}}}=-1+|\mu|^{2} \geq-1 .
$$

Now we can apply the generalized maximum principle to conclude that there is a sequence $\left\{z_{k}\right\} \in \Omega$ such that

$$
\lim _{k \rightarrow \infty} \eta\left(z_{k}\right)=\bar{\eta}=\sup \eta, \quad \limsup _{k \rightarrow \infty} \Delta_{g} \eta\left(z_{k}\right) \leq 0 .
$$

By taking a subsequence, if necessary, we can assume

$$
\lim _{k \rightarrow \infty}|\mu|^{2}\left(z_{k}\right)=a \leq 1
$$

Therefore, by taking limit as $k \rightarrow \infty$ in the above equation, we have

$$
e^{2 \bar{\eta}}-a e^{-2 \bar{\eta}}-1+a \leq 0
$$

which implies $v \leq \tilde{w}$.

Corollary 7. Let $h$ be a continuous function defined on a domain $\Omega \subset$ $\mathbf{C}$, and let $\tilde{w}_{1}$ and $\tilde{w}_{2}$ be two solutions of

$$
\Delta \tilde{w}=e^{2 \tilde{w}}-h^{2} e^{-2 \tilde{w}} \geq 0
$$

in $\Omega$ such that $e^{2 \tilde{w}_{i}}|d z|^{2}$ are complete metrics on $\Omega$ for $i=1,2$. Then $\tilde{w}_{1} \equiv \tilde{w}_{2}$.

Corollary 8. We have at most one solution of (2) such that the metric is complete.

Proof. Let $w_{i}, i=1,2$, be two solutions of (2) such that both metrics are complete. Then $\tilde{w}_{i}$, defined by

$$
e^{2 \tilde{w}_{i}}=e^{2 w_{i}} \frac{4}{\left(1-|z|^{2}\right)^{2}},
$$


are solutions of (3) with $h=|\Phi|$ the Euclidean norm of $\Phi$. Therefore, $\tilde{w}_{1} \equiv \tilde{w}_{2}$, which implies $w_{1} \equiv w_{2}$.

\section{Existence}

For the existence, we treat a special case first. As we will see later that this corresponds to those harmonic maps which are quasi-conformal. The basic theorem which we use here is the theorem on the method of suband super-solutions. Since it is not easy to find a reference for the case of complete noncompact manifolds, we include a sketch of the proof here for a version in our setting.

Theorem 9. Let $M$ be a complete Riemannian manifold, and $F(x, u)$ be a $C^{\infty}$-function on $M \times \mathbf{R}$ such that

$$
\frac{\partial F}{\partial u}>0 \quad \forall(x, u) \in M \times \mathbf{R} .
$$

If there exists $\underline{\psi} \leq \bar{\psi} \in C^{0}(M) \cap H^{1}(M)$ such that

$$
\left\{\begin{array}{l}
\Delta \bar{\psi} \leq F(x, \bar{\psi}) \\
\Delta \underline{\psi} \geq F(x, \underline{\psi})
\end{array}\right. \text { weakly, }
$$

then there exists $u \in C^{\infty}(M)$ such that $\Delta u=F(x, u)$ and $\psi \leq u \leq \bar{\psi}$, where $\psi$ and $\bar{\psi}$ are called the sub-and super-solutions respectively.

Sketch of proof. Choose a sequence of domains $\left\{D_{k}\right\}_{k=1}^{\infty}$ in $M$ such that $D_{k} \subset D_{k+1}, \bigcup_{k=1}^{\infty} D_{k}=M$, and with sufficiently regular boundary. Then choose a boundary data $\psi_{k}$ for each $D_{k}$ such that $\psi \leq \psi_{k} \leq \bar{\psi}$ on $D_{k}$. The method of sub- and super-solutions for compact domain implies that there is a $C^{\infty}$ solution $u_{k}$ on $D_{k}$ such that $\underline{\psi} \leq u_{k} \leq \bar{\psi}$.

Using the fact that $\frac{\partial F}{\partial u}>0$ and $\underline{\psi} \leq u_{k} \leq \bar{\psi}$, the interior Schauder estimate [6] yields that $\left\{u_{k+k_{0}}\right\}_{k=1}^{\infty}$ is bounded in $C^{2, \alpha}\left(D_{k_{0}}\right)$. Since $\left\{D_{k}\right\}_{k=1}^{\infty}$ is an exhaustion of $M$, we have, for any compact subset $K \subset M$,

$$
\left\|u_{k+k_{0}}\right\|_{C^{2, \alpha}(K)}<C(K)
$$

for all positive integers $k$. Hence there is a subsequence $\left\{u_{k^{\prime}}\right\}$ which converges in $C_{\text {loc }}^{2}(M)$, and the limiting function $u$ is a $C^{2}$ solution of the equation. Standard regularity theory [6] implies that $u$ is $C^{\infty}$. Since $\underline{\psi} \leq u_{k^{\prime}} \leq \bar{\psi}$, we also have $\psi \leq u \leq \bar{\psi}$, which completes our proof.

Proposition 10. Let $\Phi$ be a holomorphic quadratic differential on $\mathbf{D}$ such that $\|\Phi\|$ is bounded. Then (2) has a unique $C^{\infty}$ solution $w$ which 
satisfies

$$
1 \leq e^{2 w} \leq \frac{1+\sqrt{1+4 a^{2}}}{2}
$$

where $a=\sup \|\Phi\|$.

Proof. We shall use the method of sub- and super-solutions. First of all, we have

Claim 11. $\underline{\psi}=\frac{1}{2} \log ^{+}\|\Phi\|$ is a weak subsolution of $(2)$, where

$$
\log ^{+}\|\Phi\|= \begin{cases}\log \|\Phi\| & \text { if }\|\Phi\| \geq 1, \\ 0 & \text { if }\|\Phi\| \leq 1 .\end{cases}
$$

Proof of claim. In fact $\underline{\psi}=\sup \left\{0, \frac{1}{2} \log \|\Phi\|\right\}$, so we only need to show that $\frac{1}{2} \log \|\Phi\|$ and the constant zero are weak subsolutions of (2).

The constant zero is obviously a subsolution. For $\frac{1}{2} \log \|\Phi\|$, we notice that $\frac{1}{2} \log |\phi|$ is a weak subharmonic function. Therefore,

$$
\Delta_{p} \frac{1}{2} \log \|\Phi\|-e^{2(\log \|\Phi\| / 2)}+\|\Phi\|^{2} e^{-2(\log \|\Phi\|) / 2}+1=\frac{1}{2} \Delta_{p} \log |\phi| \geq 0
$$

weakly. This proves the claim.

It is obvious that the constant $\frac{1}{2}\left(1+\sqrt{1+4 a^{2}}\right)$ is a super-solution. So, together with the claim and the theorem on the method of sub- and super-solution, we have proved our result. q.e.d.

This proposition actually is true for all disks with any radius $R$, as long as we use the corresponding Poincaré metric $4 R^{2}|d z|^{2} /\left(R^{2}-|z|^{2}\right)^{2}$.

We now remove the restriction that $\|\Phi\|$ is bounded.

Theorem 12. Let $\Phi$ be a holomorphic quadratic differential on $\mathbf{D}$. Then (2) has a unique $C^{\infty}$ solution.

Proof. For any positive integer $k$, let

$$
D_{k}=\{z \in \mathbf{D}: d(z)<k\}=\left\{z \in \mathbf{D}:|z|<R_{k}\right\},
$$

where $d(z)=\log [(1+|z|) /(1-|z|)]$ is the Poincare distance function from the origin, and $R_{k}=\left(e^{k}-1\right) /\left(e^{k}+1\right)<1$. Then we have

$$
D_{1} \subset \cdots \subset D_{k} \subset D_{k+1} \subset \cdots \subset \mathbf{D} \text {, }
$$

and $\bigcup_{k=1}^{\infty} D_{k}=\mathbf{D}$.

By Proposition 10, for each $k$, we have a unique solution $\tilde{w}_{k}$ to the problem

$$
\begin{cases}\Delta \tilde{w}_{k}=e^{2 \tilde{w}_{k}}-|\Phi|^{2} e^{-2 \tilde{w}_{k}} \geq 0 & \text { on } D_{k} \\ \left(D_{k}, e^{2 \tilde{w}_{k}}|d z|^{2}\right) & \text { complete. }\end{cases}
$$


Moreover, $\tilde{w}_{k}$ satisfies

$$
e^{2 \tilde{w}_{k}} \geq \frac{4 R_{k}^{2}}{\left(R_{k}^{2}-|z|^{2}\right)^{2}} \geq \frac{4}{\left(1-|z|^{2}\right)^{2}} \quad \forall z \in D_{k} .
$$

Since $\left(D_{k}, e^{2 \tilde{w}_{k}}|d z|^{2}\right)$ is complete and $\tilde{w}_{k+1}$ restricted to $D_{k}$ is still a solution to the equation

$$
\Delta \tilde{w}=e^{2 \tilde{w}}-|\Phi|^{2} e^{-2 \tilde{w}},
$$

we have, from Theorem 6, $\tilde{w}_{k+1} \leq \tilde{w}_{k}$ in $D_{k}$. Hence $\tilde{w}_{k+j} \leq \tilde{w}_{k}$ in $D_{k}$ for all positive integers $j$. Using the fact that $e^{2 \tilde{w}_{k}} \geq 4 /\left(1-|z|^{2}\right)^{2}$, we see that $\tilde{w}_{k}$ converges pointwise to a function $\tilde{w}$. However, in order to complete the proof, we need to show, at least for a subsequence, that

$$
\tilde{w}_{k} \rightarrow \tilde{w} \text { in } C_{\mathrm{loc}}^{2}(\mathbf{D}) \text {, }
$$

i.e., for any compact subset $K \subset \mathbf{D}, \tilde{w}_{k}$ is defined on $K$ for all large enough $k$, and

$$
\left\|\tilde{w}_{k}-\tilde{w}\right\|_{C^{2}(K)} \rightarrow 0
$$

Since $\bigcup_{k=1}^{\infty} D_{k}=\mathbf{D}$, we only need to show this for $K=\bar{D}_{k}$. Now consider the sequence $\left\{\tilde{w}_{k+j+2}\right\}_{j=1}^{\infty}$ on $\bar{D}_{k}$. Choose a disk $B_{k}$ such that $\bar{D}_{k} \subset B_{k} \Subset$ $D_{k+1}$. Since $B_{k}$ satisfies the uniform interior cone condition, we have the Sobolev inequality

$$
\|u\|_{C^{3}\left(\bar{D}_{k}\right)} \leq\|u\|_{C^{3}\left(B_{k}\right)} \leq C\|u\|_{W^{5,2}\left(B_{k}\right)},
$$

and from the elliptic interior $L^{2}$-estimate [6] it follows that

$$
\|u\|_{W^{l+2,2}\left(\Omega^{\prime}\right)} \leq C\left[\|u\|_{W^{1,2}(\Omega)}+\|\Delta u\|_{W^{l, 2}(\Omega)}\right],
$$

where $\Omega^{\prime} \Subset \Omega$ and $C=C\left(l, \Omega^{\prime}, \Omega\right)$.

So, in order to estimate $\left\|\tilde{w}_{k+j+2}\right\|_{C^{3}\left(\bar{D}_{k}\right)}$, we only need to estimate

$$
\left\|\tilde{w}_{k+j+2}\right\|_{W^{1,2}\left(D_{k+1}\right)} \text {. }
$$

In fact

$$
\Delta \tilde{w}_{k+j+2}=e^{2 \tilde{w}_{k+j+2}}-|\Phi|^{2} e^{-2 \tilde{w}_{k+j+2}}
$$

implies

$$
\nabla\left(\Delta \tilde{w}_{k+j+2}\right)=2\left(e^{2 \tilde{w}_{k+j+2}}+|\Phi|^{2} e^{-2 \tilde{w}_{k+j+2}}\right) \nabla \tilde{w}_{k+j+2}-e^{-2 \tilde{w}_{k+j+2}} \nabla|\Phi|^{2},
$$

which in turn implies

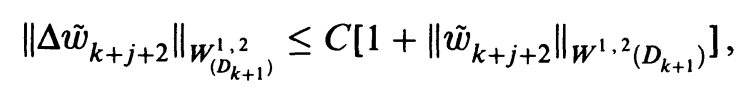


where $C=C(k)$ is independent of $j$. So, by an interior $L^{2}$-estimate and iterating the procedure, we obtain

$$
\left\|\tilde{w}_{k+j+2}\right\|_{W^{5,2}\left(\Omega^{\prime}\right)} \leq C\left(k, \Omega^{\prime}\right)\left[1+\left\|\tilde{w}_{k+j+2}\right\|_{W^{1,2}\left(D_{k+1}\right)}\right],
$$

provided $\Omega^{\prime} \Subset D_{k+1}$. Therefore, taking $\Omega^{\prime}=B_{k}$ and using the Sobolev inequality yield

$$
\left\|\tilde{w}_{k+j+2}\right\|_{C^{3}\left(\bar{D}_{k}\right)} \leq C(k),
$$

provided $\left\|\tilde{w}_{k+j+2}\right\|_{W_{\left(D_{k+1}\right)}^{1,2}} \leq C(k)$.

To estimate $\left\|\tilde{w}_{k+j+2}\right\|_{W^{1,2}\left(D_{k+1}\right)}$, we consider the equation

$$
\Delta \tilde{w}_{k+j+2}=e^{2 \tilde{w}_{k+j+2}}-|\Phi|^{2} e^{-2 \tilde{w}_{k+j+2}} \text { on } D_{k+2} \text {. }
$$

Choose a positive test function $\varphi \subset C_{0}^{\infty}\left(D_{k+2}\right)$ such that $\varphi=1$ on $D_{k+1}$. In the following calculation, we shall write $\tilde{w}$ for $\tilde{w}_{k+j+2}$. Then

$$
\int_{D_{k+2}} \varphi^{2} \tilde{w} \Delta \tilde{w}=\int_{D_{k+2}} \varphi^{2}\left(e^{2 \tilde{w}}-|\Phi|^{2} e^{-2 \tilde{w}}\right) \geq 0,
$$

which implies

$$
\int_{D_{k+2}} \nabla\left(\varphi^{2} \tilde{w}\right) \cdot \nabla \tilde{w} \leq 0 .
$$

Thus by using Hölder's inequality and integration by parts we obtain

$$
\int_{D_{k+2}} \varphi^{2}|\nabla \tilde{w}|^{2} \leq 2 \int_{D_{k+2}} \tilde{w}^{2}|\nabla \varphi|^{2},
$$

which implies, in consequence of $\varphi=1$ on $D_{k+1}$,

$$
\int_{D_{k+2}}|\nabla \tilde{w}|^{2} \leq C(k)\|\tilde{w}\|_{L^{2}\left(D_{k+2}\right)}
$$

That is,

$$
\left\|\tilde{w}_{k+j+2}\right\|_{W^{1,2}\left(D_{k+1}\right)} \leq C(k)\left\|\tilde{w}_{k+j+2}\right\|_{L^{2}\left(D_{k+2}\right)} .
$$

Since $\left\|\tilde{w}_{k+j+2}\right\|_{L^{2}\left(D_{k+2}\right)} \leq\left\|\tilde{w}_{k+3}\right\|_{L^{2}\left(D_{k+2}\right)}$, we have, for any $k$,

$$
\left\|\tilde{w}_{k+j+2}\right\|_{C^{3}\left(\bar{D}_{k}\right)} \leq C(k)
$$

for all positive integers $j$.

So, using the Arzela-Ascoli theorem and a diagonal trick, we can find a subsequence $\left\{\tilde{w}_{k^{\prime}}\right\}_{k^{\prime}=1}^{\infty}$ which converges to $\tilde{w}$ in $C_{\mathrm{loc}}^{2}(\mathbf{D})$, so that $\tilde{w}$ is a solution of

$$
\Delta \tilde{w}=e^{2 \tilde{w}}-|\Phi|^{2} e^{-2 \tilde{w}}
$$


Finally, since $e^{2 \tilde{w}_{k}} \geq 4 /\left(1-|z|^{2}\right)^{2},\left(\mathbf{D}, e^{2 \tilde{w}}|d z|^{2}\right)$ is also complete. This implies that $w$, given by $4 e^{2 w} /\left(1-|z|^{2}\right)^{2}=e^{2 \tilde{w}}$, satisfies (2). Hence our proof is complete.

\section{Criteria for quasi-conformality}

A harmonic diffeomorphism between compact Riemann surfaces is always a quasi-conformal mapping. The study of harmonic maps between compact Riemann surfaces leads to a parametrization of finite Teichmüller spaces. So it is interesting to understand the relationship between universal Teichmüller space and quasi-conformal harmonic diffeomorphisms between Poincaré disks.

In view of the result in $\S 6$, it is reasonable to study under what condition on the Hopf differential the harmonic map from $\mathbf{D}$ into itself is actually a quasi-conformal mapping. The following result gives a complete answer to this question.

Theorem 13. Let $u$ be an orientation preserving harmonic map from $\mathbf{D}$ onto itself, and let $\Phi$ be the Hopf differential of $u$. Then $u$ is quasiconformal if and only if $\sup _{z^{\prime} \in \mathbf{D}}\|\Phi\|(z)<+\infty$. In fact, the fact that $u$ has bounded dilatation suffices to imply that $u$ is a quasi-isometry of the Poincaré disk.

Proof. If $u$ is quasi-conformal, even if $u$ only has bounded dilation, then, for some $\delta>0$,

$$
e^{2 w}-\|\Phi\|^{2} e^{-2 w}=e^{2 w}\left(1-|\mu|^{2}\right)>\delta e^{2 w} .
$$

So

$$
\Delta_{p} w \geq \delta e^{2 w}-1
$$

and the maximum principle implies $e^{2 w} \leq \delta^{-1}$. Since $\|\Phi\| \leq e^{2 w}$, we have sup $\|\Phi\|<+\infty$. Moreover, together with $1 \leq e^{2 w}$, we conclude that $u$ is a quasi-isometry of the Poincare disk.

Conversely, if $\sup \|\Phi\|$ is bounded, then by the maximum principle,

$$
1 \leq e^{2 w} \leq \frac{1}{2}\left[1+\sqrt{1+4(\sup \|\Phi\|)^{2}}\right]<C
$$

for some fixed $C$. So $\left(\mathbf{D}, e^{2 w} d s_{p}^{2}\right)$ is equivalent to $\left(\mathbf{D}, d s_{p}^{2}\right)$. This implies that $\left(\mathbf{D}, e^{2 w} d s_{p}^{2}\right)$ has exponential volume growth.

Let $B_{r}(x)$ be a geodesic ball in $\mathbf{D}$ with radius $r$ and center $x$, and let $L_{r}(x)$ be the length of $\partial B_{r}(x)$. Then $\frac{d}{d r} L_{r}(x)=\int_{\partial B_{r}(x)} k_{g}$, where $k_{g}$ 
is the geodesic curvature of $\partial B_{r}(x)$. But $\frac{d}{d r} V\left(B_{r}\right)=L_{r}$, where $V\left(B_{r}\right)$ is the volume of $B_{r}$, therefore

$$
\frac{d^{2}}{d r^{2}} V\left(B_{r}\right)=\int_{\partial B_{r}} k_{g}
$$

The Gauss-Bonnet theorem now yields

$$
\frac{d^{2}}{d r^{2}} V\left(B_{r}\right)-\pi=\int_{B_{r}(x)}-K
$$

Since $V\left(B_{x}\right)$ has exponential growth, we have, for any $\varepsilon>0$, a large enough $R>0$ such that for any $x \in \mathbf{D}$ there exists some $r, 0<r<R$, such that

$$
\frac{d^{2}}{d r^{2}} V\left(B_{r}\right)>\pi+\varepsilon,
$$

so that

$$
\varepsilon \leq \int_{B_{r}(x)}-K .
$$

A straightforward calculation gives

$$
\Delta_{g}(-K) \leq 2(-K) .
$$

Since $e^{2 w} d s_{p}^{2}$ is complete, has exponential volume growth, and its curvature is bounded from below, the super-mean value inequality (see [5])

$$
\left(\int_{B_{r}(x)}-K\right)^{1 / p} \leq C[-K(x)]
$$

holds for some $C$ and $0<p<1$ independent of $x$. Therefore, $K(x) \leq$ $-\delta$ for some $\delta>0$, which implies

$$
|\mu|^{2}=1+K \leq 1-\delta .
$$

Hence, $u$ is quasi-conformal.

Remark. It then follows from this theorem that a harmonic diffeomorphism between Poincare disks has bounded energy density if and only if it is a quasi-conformal mapping. L. F. Tam pointed out to us that this statement may have higher dimensional generalization while holomorphic quadratic differential is only defined for dimension two.

\section{Universal Teichmüller space}

Universal Teichmüller space has at least three equivalent definitions. It is most convenient for us to prove our result by using the complex 
characteristic of quasi-conformal maps between disks to define the universal Teichmüller space.

Let $\mathscr{M}$ be the set of all bounded measurable functions $\mu$ defined on the unit disk with $\|\mu\|_{\infty}<1$. Then for any $\mu \in \mathscr{M}$, there exists unique $f_{\mu}: \mathbf{C} \rightarrow \mathbf{C}$, such that $f_{\mu}$ has complex characteristic equal to $\mu$ inside $\mathbf{D}$, and is conformal outside $\mathbf{D}$ with fixed points $1, i,-1$. We say that $\mu \sim \nu$ for $\mu, \nu \in \mathscr{M}$ if

$$
\left.f_{\mu}\right|_{\partial \mathbf{D}}=\left.f_{\nu}\right|_{\partial \mathbf{D}}
$$

Then the universal Teichmüller space $T$ can be defined as $\mathscr{M} / \sim$.

We also have Teichmüller metric on $T$. Let $p, q \in T$; then the Teichmüller metric is given by

$$
d(p, q)=\inf _{\mu \in p, \nu \in q}\left\{\log \frac{1+\|(\mu-\nu) /(1-\bar{\nu} \mu)\|_{\infty}}{1-\|(\mu-\nu) /(1-\bar{\nu} \mu)\|_{\infty}}\right\}
$$

Moreover, $T$ is path-connected.

From the above definition of $T$ and the result in $\S 7$, we see that for any $\Phi \in \mathrm{BQD}=\{\Phi \in Q D:\|\Phi\|<+\infty\}$, we can associate an element [ $\mu$ ] in $T$, where $\mu$ is the complex characteristic of the quasi-conformal harmonic diffeomorphism associated to $\Phi$ which fixes $1, i$, and -1 . We denote this map by $\mathscr{B}$. Then we have the following result about $\mathscr{B}$ :

Proposition 14. $\mathscr{B}: \mathrm{BQD} \rightarrow T$ is continuous.

Proof. Let $\Phi_{1}$ and $\Phi_{2}$ be two elements in BQD, and let $p_{i}=\mathscr{B}\left(\Phi_{i}\right) \in$ $T$. Then the complex characteristics $\mu_{i}$ associated to $\Phi_{i}$ are representatives for $p_{i}$. By the definition of $d\left(p_{1}, p_{2}\right)$, we only need to show

$$
\left\|\mu_{1}-\mu_{2}\right\|_{\infty} \rightarrow 0 \text { as }\left\|\mid \Phi_{1}-\Phi_{2}\right\| \| \rightarrow 0,
$$

where $\|\mid \Phi\|\|=\sup \| \Phi \|$.

Consider the equations

$$
\Delta_{p} w_{i}=e^{2 w_{i}}-\left\|\Phi_{i}\right\|^{2} e^{-2 w_{i}}-1
$$

Let $\eta=w_{1}-w_{2}$; then

$$
\begin{aligned}
e^{-2 w_{2}} \Delta_{p} \eta= & e^{-2 \eta}-\left|\mu_{2}\right|^{2} e^{-2 \eta}-1+\left|\mu_{2}\right|^{2} \\
& +\left(\left\|\Phi_{1}\right\|-\left\|\Phi_{2}\right\|\right)\left(\left|\mu_{1}\right| e^{-2 w_{2}}+\left|\mu_{2}\right| e^{-2 w_{1}}\right) .
\end{aligned}
$$

If $\left\|\mid \Phi_{1}-\Phi_{2}\right\|<\varepsilon$, then

$$
e^{-2 w_{2}} \Delta_{p} \eta \geq e^{2 \eta}-e^{-2 \eta}-1+2 \varepsilon
$$

and the maximum principle implies, for some $0 \leq a \leq 1$,

$$
e^{2 \eta} \leq \frac{1}{2}\left[1-a^{2}+2 \varepsilon+\sqrt{\left(1-a^{2}+2 \varepsilon\right)^{2}+4 a^{2}}\right],
$$


which tends to 1 as $\varepsilon \rightarrow 0$. So

$$
\limsup _{\left\|\Phi_{1}-\Phi_{2}\right\| \| \rightarrow 0}\left(w_{1}-w_{2}\right) \leq 0
$$

Similarly, the above inequality with $w_{1}$ and $w_{2}$ interchanged also holds. Hence $\left\|w_{1}-w_{2}\right\| \rightarrow 0$ as $\left\|\Phi_{1}-\Phi_{2}\right\| \| \rightarrow 0$. This implies $\left\|\mu_{1}-\mu_{2}\right\| \rightarrow 0$ as $\left\|\mid \Phi_{1}-\Phi_{2}\right\| \| \rightarrow 0$. q.e.d.

We end our discussion with the following question. Is $\mathscr{B}$ a homeomorphism between BQD and $T$ ? This question is equivalent to the existence and uniqueness of the ideal boundary value problem for a harmonic map between Poincaré disks with quasi-symmetric boundary data.

\section{References}

[1] K. Akutagawa, Harmonic diffeomorphisms of the hyperbolic plane, preprint.

[2] K. Akutagawa \& S. Nishikawa, The Gauss map and spacelike surface with prescribed mean curvature in Minkowski 3-space, Tôhoku Math. J. 42 (1990) 67-82.

[3] S. Y. Cheng \& S. T. Yau, Differential equations on Riemannian manifolds and their geometric applications, Comm. Pure. Appl. Math. 28 (1975) 333-354.

[4] __ Maximal spacelike hypersurfaces in the Lorentz-Minkowski space, Ann. of Math. (2) 104 (1976) 407-419.

[5] H. I. Choi \& A. Treibergs, Gauss maps of spacelike constant mean curvature hypersurfaces of Minkowski space, J. Differential Geometry 32 (1990), 775-817.

[6] D. Gilbarg \& N. S. Trudinger, Elliptic partial differential equations of second order, 2nd ed., Springer, New York, 1983.

[7] T. K. Milnor, Harmonic maps and classical surface theory in Minkowski space, Trans. Amer. Math. Soc. 280 (1983) 161-185.

[8] R. Schoen \& S. T. Yau, On univalent harmonic maps between surfaces, Invent. Math. 44 (1978) 265-278.

[9] M. Wolf, The Teichmüller theory of harmonic maps, J. Differential Geometry 29 (1989) 449-479. 
